\title{
Psicooncología
}

ISSN: 1696-7240

\section{Análisis de la comunicación intra-familiar de los resultados genéticos diagnósticos en cáncer hereditario}

\author{
Cristina del Barrio ${ }^{1}$; Miguel del Campo²; Luis Pérez-Jurado 3 ; Alexandre Teulé4; Sílvia \\ Iglesias $^{5}$; Mònica Salinas ${ }^{6}$; Ignacio Blanco ${ }^{7 *}$
}

Recibido: 11 de noviembre de 2016 / Aceptado: 15 de abril de 2017

Resumen. A lo largo de la consulta de Asesoramiento Genético se hace un gran énfasis en la necesidad de comunicar la información del riesgo familiar y del estudio genético a los familiares. Además, los informes clínicos especifican los familiares en situación de riesgo. Sin embargo, desconocemos el patrón de comunicación intra-familiar de los resultados genéticos diagnósticos tras el asesoramiento.

Objetivo: Realizar un estudio descriptivo sobre el patrón de comunicación de resultado del estudio genético diagnóstico en predisposición hereditaria al cáncer en la Unidad de Asesoramiento Genético del ICO.

Método: Se ha realizado un estudio descriptivo mediante entrevista telefónica a una muestra de casos índice atendidos en la Unidad de Asesoramiento Genético que recibieron el resultado de un diagnóstico genético, explorando a qué familiares han comunicado estos resultados (patrón de comunicación familiar). Del mismo modo, se han recogido variables demográficas, personales y del propio resultado genético, para explorar si alguna de ellas pudiera modificar el patrón de comunicación.

Resultados: La mayoría de los pacientes comunican los resultados de los estudios genéticos a sus familiares. Sin embargo, esta comunicación no es completa, por lo que es posible diseñar estrategias de intervención que mejoren el patrón de comunicación de los pacientes que reciben estudios genéticos diagnósticos en el contexto de la predisposición hereditaria al cáncer.

Palabras clave: Asesoramiento genético; cáncer hereditario; patrón de comunicación familiar.

\section{[en] Analysis of intra-familial communication of diagnostic genetic results in hereditary cancer}

1 Máster en Asesoramiento Genético, Universitat Pompeu Fabra, Barcelona.

E-mail: cristina.delbarrio.gascon@gmail.com

2 Hospital Germans Trias i Pujol, Barcelona.

migueldelcampocasanelles@gmail.com

3 Hospital Germans Trias i Pujol, Barcelona.

E-mail: luis.perez@upf.edu

4 Institut Català d'Oncologia (ICO), Hospital Duran i Reynals, Hospital Germans Tries i Pujol, Barcelona. E-mail: ateule@iconcologia.net

$5 \quad$ Institut Català d'Oncologia (ICO), Hospital Duran i Reynals, Hospital Germans Tries i Pujol, Barcelona. E-mail: siglesias@iconcologia.net

6 Institut Català d'Oncologia (ICO), Hospital Duran i Reynals, Hospital Germans Tries i Pujol, Barcelona. E-mail: msalinas@iconcologia.net

7 Institut Català d'Oncologia (ICO), Hospital Duran i Reynals, Hospital Germans Tries i Pujol, Barcelona. E-mail: iblanco.germanstrias@gencat.cat

* Dirección de correspondencia: Dr. Ignacio Blanco. Coordinador - Programa de Asesoramiento y Genética Clínica. Hospital Germans Trias | Gerència Metropolitana Nord | Institut Català de la Salut. Hospital Universitari Germans Trias i Pujol | Ctra. de Canyet, s/n | 08916 Badalona. E-mail: iblanco.germanstrias@gencat.cat 
Abstract. Throughout the Genetic Counselling process a great emphasis is done on the need to communicate the familial risk information and the genetic study to the relatives. In addition, the clinical reports specify the relatives at risk situation. However, the familial communication pattern of genetic results after the counselling remains unknown.

Objective: To conduct a descriptive study about the communication pattern of results of the diagnostic genetic test in hereditary predisposition to cancer at the ICO Genetic Counselling Unit.

Methods: A descriptive study has been performed by telephone interview on a sample of index individuals attended at the Genetic Counselling Unit. Patients were asked whether if they had communicated their genetic study results and to whom. Similarly, demographic, personal and genetic result itself variables have been collected to explore whether any of them could modify the communication pattern.

Results: Most patients report the results of the genetic studies to their relatives. However, this communication is not complete, so it is possible to design intervention strategies which may improve the communication pattern of the patients who receive diagnostic genetic tests in the context of the hereditary predisposition to cancer.

Keywords: Genetic counselling; hereditary cancer; familial communication pattern.

Sumario. 1. Introducción 2. Objetivos 3. Material y métodos 3.1. Diseño 3.2. Sujetos de estudio 3.3. Variables 3.4. Recogida y análisis de datos 3.5. Modelo de entrevista telefónica 3.6. Consideraciones éticas 4. Resultados 5. Discusión 6. Agradecimientos 7. Referencias bibliográficas.

Cómo citar: Del Barrio C, Del Campo M, Pérez L, Teulé T, Iglesias S, Salinas M, Blanco I. Análisis de la comunicación intra-familiar de los resultados genéticos diagnósticos en cáncer hereditario Psicooncologia 2017; 14: 41-52. DOI: 10.5209/PSIC.55810

\section{Introducción}

El cáncer es una de las enfermedades más comunes y aparece con mayor frecuencia a edades avanzadas, como consecuencia de alteraciones genéticas producidas a lo largo de la vida bajo la influencia de factores ambientales, entre otros. A pesar de ello, los recientes avances en Genética Molecular han comportado la identificación de diversos genes de predisposición de algunos tipos de cánceres. Hoy en día, los estudios genéticos van dirigidos, principalmente, hacia 3 síndromes de cáncer familiar: Síndrome de Cáncer de Mama y Ovario Hereditario, Síndrome de Cáncer Colo-rectal Hereditario y Poliposis Adenomatosa Familiar. Así mismo, existen otros síndromes de cáncer hereditario, como son las Neoplasias Endocrinas Múltiples (NEM I y II), la Enfermedad de von Hippel-Lindau, el Síndrome de Li-Fraumeni y $\operatorname{otros}^{(1)}$.

El Asesoramiento Genético es el proceso que tiene como objetivo ayudar a entender y adaptarse a las consecuencias médicas, psicológicas y familiares de una determinada condición genética. En el caso particular del Asesoramiento Genético en predisposición hereditaria al cáncer, el asesor genético deberá informar y comunicar de forma no-directiva de la probabilidad de presentar o transmitir a su descendencia una determinada susceptibilidad genética a desarrollar una neoplasia, sobre sus implicaciones, sobre la posibilidad de realizar un diagnóstico molecular y sobre cuáles son las medidas disponibles para la prevención y el diagnóstico precoz ${ }^{(1)}$.

El proceso de valoración del riesgo puede incluir o no la realización de un estudio genético. El estudio genético es un instrumento de ayuda para la valoración del riesgo una vez que, por criterios clínicos, se considera alto. Son estos los casos donde se recomienda el Asesoramiento Genético(1). 
Los resultados del estudio genético diagnóstico en el caso índice de una familia, cuando no se conoce previamente la mutación responsable de la susceptibilidad genética, pueden ser de 2 tipos: mutación patogénica encontrada (considerando estos resultados como Positivos) o mutación no encontrada o variante de significado incierto (considerando estos resultados como No-informativos) ${ }^{(1)}$.

La comunicación del resultado genético diagnóstico del caso índice a los familiares es un elemento crucial dentro del proceso de Asesoramiento Genético para identificar qué familiares se pueden beneficiar de realizar un estudio genético y que, de este modo, puedan adherirse a las medidas de cribado recomendadas ${ }^{(2)}$.

En el proceso de Asesoramiento Genético en predisposición hereditaria al cáncer, el individuo índice o caso índice que se somete al estudio genético diagnóstico se convierte en el nexo de unión entre la Unidad de Asesoramiento Genético y el resto de la familia. El individuo índice, o quien él decida, será el responsable de comunicar la evaluación del riesgo realizada en la Unidad, las estrategias preventivas recomendadas y, por último, los resultados del estudio genético al resto de la familia ${ }^{(3,4)}$.

El patrón de comunicación de resultado genético del caso índice a su familia influye en que los familiares en riesgo acudan o no a la Unidad de Asesoramiento Genético en cáncer hereditario.

\section{Objetivos}

Los objetivos del presente análisis son: Describir el patrón de comunicación familiar del estudio genético diagnóstico en cáncer hereditario tras la consulta en la Unidad de Asesoramiento Genético; así como describir las variables demográficas y personales (grado de parentesco, sexo, edad, profesión, nivel de estudios) en el patrón de comunicación familiar del cáncer hereditario tras la consulta en la Unidad de Asesoramiento Genético.

\section{Material y métodos}

\subsection{Diseño}

Se realiza un estudio descriptivo sobre el patrón de comunicación familiar del resultado de un estudio genético diagnóstico (positivo o no informativo [indeterminado o Variante de Significado Incierto]).

\subsection{Sujetos de estudio}

Los sujetos de estudio son 100 casos índice a los que se les ha entregado el resultado de un estudio genético diagnóstico en cáncer hereditario de mama y colon desde Enero de 2009 en la Unidad de Asesoramiento Genético del Institut Català d'Oncologia (ICO), Hospital Universitari de Bellvitge, Barcelona. 


\subsection{Variables}

Se exploran las variables: "Edad", "Sexo", "Nivel de estudios", "Profesión", "Patología de base", "Resultado del estudio genético", "Grado de comprensión", "Familiares a los que debería informar", "Familiares a los que ha informado", "Canal de comunicación con los familiares", "Motivos por los que no ha informado a alguno de sus familiares".

Para crear la variable de cálculo "¿Informados todos los familiares que debían ser informados?", se ha superpuesto la variable "Familiares que debían ser informados" con la variable "Familiares informados". En caso de coincidencia de todos los familiares, el valor de la nueva variable es "Sí"; en el caso que algún familiar que debía ser informado no lo hubiese sido, el valor es "No".

\subsection{Recogida y análisis de datos}

Se recogen y analizan las historias clínicas y los informes de los casos índice. Se realiza una entrevista telefónica a los casos índice para determinar el patrón de comunicación familiar, sin intervención.

\subsection{Modelo de entrevista telefónica}

En la entrevista telefónica, la Investigadora se presenta como un miembro de la Unidad de Asesoramiento Genético del ICO, se enuncia el tema del Proyecto de Investigación ("Comunicación de Resultados Genéticos a Familiares") y se pregunta a los encuestados:"¿A qué familiares han comunicado la información de su estudio genético y del riesgo?”; “CCómo han comunicado la información (personalmente, por carta o por teléfono)?”; “¿A qué familiares no han informado y por qué motivos?"; "En una escala del 1 al 10, ¿cómo comprendieron el resultado genético y el riesgo para poder transmitir correctamente la información a los familiares?”; “Cuál es su profesión y su nivel de estudios?”; si tiene alguna duda, se ofrece hablar con alguna enfermera de la Unidad y, por último, se agradece su colaboración.

\subsection{Consideraciones éticas}

Los pacientes a entrevistar telefónicamente habían concedido previamente su consentimiento para ser contactados por parte de la Unidad de Asesoramiento Genético en un futuro, por lo que no se vulnera el principio ético de privacidad. $\mathrm{Al}$ inicio de la entrevista se solicita la participación voluntaria en la misma. No se revelan datos sobre qué miembros de la familia acuden a la Unidad, sólo se pregunta a quién comunican el resultado del estudio genético y cómo.

\section{Resultados}

La muestra de casos índice entrevistados telefónicamente es de 100 individuos $(\mathrm{N}=$ 100). La muestra se divide en 39 casos diagnosticados de cáncer de colon y 61, de cáncer de mama. 
En la tabla 1 se indica el número de casos índice analizados en función del sexo, de la edad, de la profesión y del nivel de estudios.

El 19\% de los casos índices son hombres; mientras que el 81\%, mujeres.

Respecto a la edad, destaca: una ausencia de casos menores de 25 años y un $63 \%$, en la franja entre 46 y 65 años.

El 53\% de los pacientes entrevistados trabaja fuera de su domicilio en la actualidad: un $1 \%$ se dedica a la agricultura y a la ganadería; un $1 \%$, a la industria; mientras que un $51 \%$, a los servicios.

Respecto al nivel de estudios, destaca: un $2 \%$ tiene un Doctorado; mientras que un $41 \%$, estudios primarios.

En la tabla 2 se muestra el número de casos índice analizados en función del tipo de resultado y de la comprensión del resultado.

Respecto al tipo de resultado, el $75 \%$ de los casos índice ha obtenido un resultado genético diagnóstico no informativo; mientras que el $25 \%$, un resultado positivo.

Tabla 1. $\mathrm{N}^{\mathrm{o}}$ de casos índice analizados en función del sexo, de la edad, de la profesión y del nivel de estudios.

\begin{tabular}{|c|c|c|c|c|}
\hline & & COLON & МАМА & TOTAL \\
\hline \multirow{2}{*}{ SEXO } & Hombre & 18 & 1 & 19 \\
\hline & Mujer & 21 & 60 & 81 \\
\hline \multirow{6}{*}{ EDAD } & $<25$ años & 0 & 0 & 0 \\
\hline & 25-35 años & 3 & 7 & 10 \\
\hline & 36-45 años & 2 & 14 & 16 \\
\hline & 46-55 años & 15 & 17 & 32 \\
\hline & 56-65 años & 14 & 17 & 31 \\
\hline & $>65$ años & 5 & 6 & 11 \\
\hline \multirow{5}{*}{ PROFESIÓN } & No trabajo & 15 & 16 & 31 \\
\hline & Ama de casa & 4 & 12 & 16 \\
\hline & Agricultura y Ganadería & 0 & 1 & 1 \\
\hline & Industria & 0 & 1 & 1 \\
\hline & Servicios & 20 & 31 & 51 \\
\hline \multirow{5}{*}{$\begin{array}{l}\text { NIVEL DE } \\
\text { ESTUDIOS }\end{array}$} & No estudios & 2 & 3 & 5 \\
\hline & Primarios & 20 & 21 & 41 \\
\hline & Secundarios & 11 & 26 & 37 \\
\hline & Diplomatura/Licenciatura & 6 & 9 & 15 \\
\hline & Doctorado & 0 & 2 & 2 \\
\hline
\end{tabular}

Destaca que todos los pacientes entrevistados refieren haber comprendido el resultado del estudio genético. Ningún caso valora la comprensión por debajo del 5. Un 64\%, la valora con una puntuación de 9 ó superior. 
Tabla 2. $\mathrm{N}^{\mathrm{o}}$ de casos índice analizados en función del tipo de resultado y de la comprensión del resultado.

\begin{tabular}{|c|c|c|c|c|}
\cline { 3 - 5 } \multicolumn{2}{c|}{} & COLON & MAMA & TOTAL \\
\hline \multirow{2}{*}{$\begin{array}{c}\text { TIPO DE } \\
\text { RESULTADO }\end{array}$} & No informativo & 23 & 52 & 75 \\
\cline { 2 - 5 } & Positivo & 16 & 9 & 25 \\
\cline { 2 - 5 } & $<5$ & 0 & 0 & 0 \\
\cline { 2 - 5 } & 5 & 0 & 3 & 3 \\
\cline { 2 - 5 } COMPRENSIÓN \\
\cline { 2 - 5 } $\begin{array}{c}\text { DEL RESULTADO } \\
(1 \text { a 10) }\end{array}$ & 6 & 3 & 1 & 4 \\
\cline { 2 - 5 } & 7 & 7 & 20 & 27 \\
\cline { 2 - 5 } & 9 & 22 & 24 & 46 \\
\hline
\end{tabular}

En la figura 1 se indican los casos índice que informan del resultado genético a sus familiares y los casos índice que no informan a los mismos.

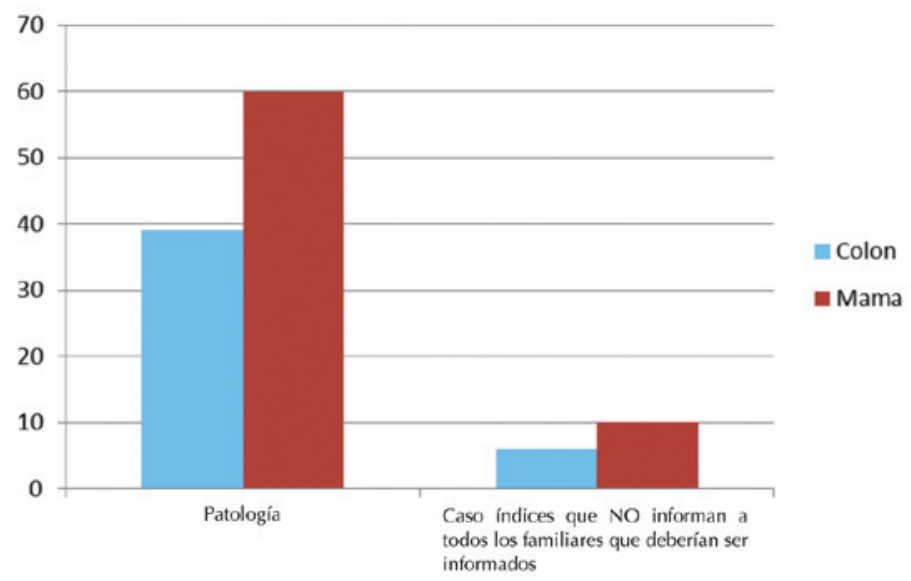

Figura 1. Porcentaje de los casos índice que no informa a todos sus familiares vs patología.

De los 100 casos índice, 99 informan de sus resultados; mientras que sólo uno decide no informar a sus familiares, pues no lo considera necesario.

Partiendo de los 99 casos índice que comunican los resultados a sus familiares, se analiza si éstos informan a todos los familiares que debían ser informados.

En la figura 2 se muestra el número de casos índice analizados en función de si han sido informados todos los familiares que debían ser informados.

Respecto a si son informados todos los familiares que debían ser informados, el $83 \%$ de los casos sí informaron a todos los familiares que deberían haber sido informados. El 16\% de los casos se dejaron a algún familiar por informar. Cabe destacar que se omite la información a 6 Familiares de Primer Grado (FPG). 


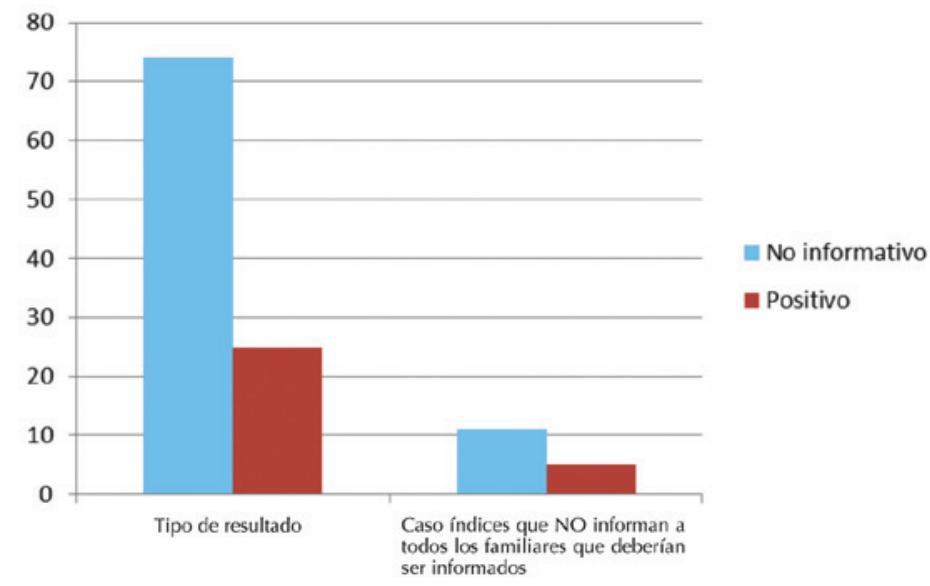

Figura 2. Porcentaje de los casos índice que no informa a todos sus familiares vs tipo de resultado.

Tras obtener el dato del 16\%, se analiza si la no-información tiene alguna relación con el hecho de padecer cáncer de mama o de colon:

- 10 casos de mama que no informan a todos sus familiares / 60 casos de mama totales $=17 \%[3 \mathrm{FPG}]$.

- 6 casos de colon que no informan a todos sus familiares / 39 casos de colon totales $=15 \%$ [3 FPG].

En la Figura 1 se muestra el porcentaje de los casos índice que no informa a todos sus familiares vs la patología (colon/mama).

Los porcentajes obtenidos no indican diferencias significativas en función de la patología.

Posteriormente, se analizó si la no-información tenía alguna relación con el tipo de resultado:

- 5 casos con resultado positivo que no informan a todos sus familiares / 25 casos con resultado positivo $=20 \%[1 \mathrm{FPG}]$.

- 11 casos con resultado no informativo que no informan a todos sus familiares / 74 casos con resultado no informativo $=15 \%[5$ FPG $]$.

En la Figura 2 se muestra el porcentaje de los casos índice que no informa a todos sus familiares vs el tipo de resultado (no informativo / positivo).

Los porcentajes obtenidos no indican diferencias significativas en función del tipo de resultado.

Los pacientes omiten información a otros familiares por diversas razones: informan otros miembros, costarles hablar de estos temas, ser miembros masculinos (en caso de cáncer de mama), no percibir interés por parte del receptor, no haberlo creído necesario, no tener relación o hallarse los familiares en paradero desconocido. 


\section{Discusión}

Se ha analizado el patrón de comunicación familiar del test genético diagnóstico en cáncer hereditario y las variables demográficas y personales de 100 casos índice (39 de colon y 61 de mama). Los resultados del análisis indican que el perfil del caso índice es una mujer de 46 a 65 años, que se dedica al sector servicios y con un nivel de estudios primarios. 99 casos índice informan a sus familiares del resultado, predominantemente por canal oral. De éstos, 83 casos índice informan a todos los familiares que debían ser informados mientras que 16 casos índice se dejan a algún familiar sin informar, entre los que destacan 6 FPG. Estas diferencias no son debidas ni al tipo de patología (cáncer de colon o cáncer de mama), ni al tipo de resultado (positivo o no informativo). Existen diferentes motivos que llevan a los casos índice a no informar a sus familiares.

El análisis de la comunicación intra-familiar de los resultados genéticos diagnósticos en la Unidad de Asesoramiento Genético en Cáncer Hereditario del ICO indica que la gran mayoría de los casos índice informan a todos sus FPG y Familiares de Segundo Grado (FSG), así como que valoran muy positivamente la comprensión del resultado genético y del riesgo familiar.

Diferentes estudios de la literatura han demostrado que la mayoría de los pacientes sometidos a pruebas genéticas comparten sus resultados con los FPG. Sin embargo, estos resultados llegan a los parientes más lejanos con menor frecuencia ${ }^{(5)}$. Los resultados obtenidos en este análisis coinciden en que la mayoría de pacientes comparten sus resultados genéticos diagnósticos con los FPG. Sin embargo, a diferencia de estudios anteriores, la mayoría de pacientes también comparten sus resultados con los FSG.

En la Literatura, se han descrito diferentes variables que pueden influir en el patrón de comunicación de los estudios genéticos. Se ha sugerido que es más probable discutir los contenidos del Asesoramiento Genético y los resultados del estudio genético diagnóstico entre dos miembros de la familia cuando: a) se ha identificado una mutación patogénica; b) si se trata de un cónyuge o un FPG; c) si no se trata de un FPG, pero donde haya relación de cohesión positiva, relación de liderazgo o relación sin conflictos ${ }^{(6)}$.

En contra de lo sugerido en estudios previos, respecto a que el estudio genético se comunica más en caso de ser positivo, los resultados obtenidos indican que no hay diferencias en la comunicación de resultados positivos y no informativos. Del análisis también se desprende que no existen diferencias en la comunicación de resultados según el tipo de patología (cáncer de colon o cáncer de mama) ${ }^{(6)}$.

El perfil del caso índice del análisis es una mujer de 46 a 65 años, que se dedica al sector de servicios y con un nivel de estudios primarios. Según la Literatura, las madres tienden a ser las personas más influyentes cuando se habla de riesgos con miembros de la familia ${ }^{(6)}$. El nivel de necesidad de información por parte de las madres se asocia a la motivación de realizar pruebas genéticas, la toma de decisiones de seguimiento y el conflicto de decisión ${ }^{(7)}$.

Los pacientes llamados refieren haber comprendido los resultados. Casi todos los casos índice informan a sus familiares del resultado, predominantemente por canal oral. De éstos, la mayoría de los casos índice informan a todos los familiares que debían ser informados. 
Los motivos que llevan a los casos índice a no informar a sus familiares son debidas a: a) la información es proporcionada por parte de otros miembros; b) la dificultad de hablar de estos temas; c) ser miembros masculinos (en el caso del cáncer de mama); d) no recibir interés por parte del receptor; e) no creer necesaria la información; f) no tener relación con sus familiares; g) hallarse sus familiares en paradero desconocido.

Existen dos limitaciones destacables en este estudio: El tamaño de la muestra ( $\mathrm{N}$ $=100$ ) y la subjetividad de los datos, ya que no se pueden confirmar las declaraciones de los pacientes en las entrevistas telefónicas.

Una propuesta sobre cómo continuar la línea de investigación para mejorar la atención en la Unidad de Asesoramiento Genético en Cáncer Hereditario sería analizar el impacto de las variables demográficas y personales (grado de parentesco, sexo, edad, profesión, nivel de estudios) en el patrón de comunicación familiar del cáncer hereditario tras la consulta en la Unidad de Asesoramiento Genético; así como las relaciones intra-familiares (cohesión positiva, liderazgo o falta de conflicto).

Un mayor conocimiento del patrón de comunicación de los estudios genéticos complementará el análisis realizado por la Unidad de Asesoramiento Genético, permitiendo diseñar y evaluar intervenciones específicas para mejorar la atención global de las familias con sospecha de predisposición hereditaria al cáncer.

A nivel global, la comunicación de la información genética dentro de las familias se puede mejorar mediante la prestación de un mayor apoyo al Asesoramiento Genético ${ }^{(8)}$. Algunos autores sugieren que los servicios clínicos podrían proporcionar un enfoque proactivo notificando a los familiares, que no son sus pacientes, la disponibilidad de pruebas genéticas sin comprometer a los principios de privacidad y autonomía ${ }^{(9)}$.

En conclusión, la mayoría de los pacientes comunican los resultados de los estudios genéticos a sus familiares. Sin embargo, esta comunicación no es completa, por lo que es posible diseñar estrategias de intervención que mejoren el patrón de comunicación de los pacientes que reciben estudios genéticos diagnósticos en el contexto de la predisposición hereditaria al cáncer.

Las últimas revisiones del tema en la Literatura se dirigen a cómo las familias comunican la información genética, a los retos de la comunicación de la información, a los factores que pueden afectar a la comunicación y a qué influencia tiene esto en las relaciones familiares.

Los resultados muestran que los casos índice dan a conocer que la responsabilidad hacia las generaciones futuras, el género, la proximidad y la falta de contacto juegan un papel fundamental en la comunicación familiar. Los medios de comunicación se citan como una fuente de información de la información genética, que tiende a tener un efecto positivo en las familias. Los resultados indican que los individuos están dispuestos a informar a los miembros de la familia, sobre todo cuando hay niños y nietos en riesgo y que se utilizan diferentes estrategias. Por otra parte, la comprensión de la Genética está regulada no sólo por sus familias, sino por la forma en la que la sociedad maneja la información. Por lo tanto, los profesionales del área de la Genética deben tener en cuenta la influencia familiar en las personas y su decisión de asistir a los servicios de Genética y también la influencia de los medios de comunicación ${ }^{(17)}$.

Resulta crítico evaluar el nivel de preparación del miembro de la familia respecto al manejo del estrés psicológico asociado al resultado genético, a la hora de 
comunicar su resultado a sus familiares. Los profesionales sanitarios deben mejorar las estrategias de manejo con el fin de promover la vida familiar normal. Esto se puede hacer inspirando actitudes positivas y de potenciación dirigidas a cuidar y a ayudar a la familia para construir las capacidades de comunicación necesarias con el fin de clarificar el resultado genético.

Existen guías clínicas para los profesionales de la Salud que trabajan con familias dentro del primer año después del diagnóstico. Los profesionales sanitarios deben escuchar y aceptar las emociones de shock por parte de los miembros de la familia que se enfrentan a un trastorno en sus vidas y a la interrupción de sus planes de futuro. Los profesionales de la Salud deben proporcionar información clara a toda la familia en relación a la comunicación de los resultados y a las estrategias de manejo familiar del resultado genético; así como estrategias para ayudar a la normalización y al retorno a su anterior estilo de vida. El equipo médico debe exhibir profesionalidad y habilidades con el fin de aumentar la confianza y la sensación de seguridad de los familiares en el entorno de la atención médica. Alentar a los miembros de la familia para desarrollar el pensamiento positivo y hacer planes para su vida futura debe ser una prioridad del plan $^{(18)}$.

Las publicaciones más recientes en la Literatura, del año 2016, proporcionan datos importantes sobre cómo las familias se comunican acerca de los resultados genéticos y las razones de si las pruebas se quieren realizar o no. Esta información puede ser utilizada por los Asesores Genéticos en el seguimiento de las familias después de un diagnóstico genético ${ }^{(19)}$.

Los hallazgos sugieren que las actitudes están más influenciadas por las características de la enfermedad que por el riesgo estadístico. En general, los encuestados expresan actitudes firmes que demuestran que una condición Genética familiar no se trata de una cuestión que los familiares consideren ambivalente ${ }^{(20)}$.

La angustia al oír el resultado de la prueba es mayor para aquellos familiares cuyo probando recibe resultados informativos. Los familiares informan de una disminución en la percepción del riesgo de cáncer después de escuchar los resultados de la prueba, independientemente del tipo de resultado. La intención de recurrir al Asesoramiento es baja, incluso entre los familiares cuyo probando recibe resultados informativos. Los familiares varones tienen menos probabilidades de ser informados de los resultados de las pruebas y más probabilidades de olvidar los mismos. Estos hallazgos sugieren formas de mejorar el proceso de comunicación dentro de las familias ${ }^{(21)}$.

Los resultados de los Estudios más recientes sugieren que los pacientes con alta preocupación por los riesgos genéticos, con bajo interés en la información genética o con un resultado negativo de la prueba genética probablemente necesitarán apoyo adicional para fomentar la comunicación familiar ${ }^{(22)}$.

En referencia al ámbito Legislativo, en Francia existe Legislación en materia de comunicación de Resultados Genéticos en la Práctica Clínica. Sería objeto de debate la introducción de Legislación en España, en el ámbito de la comunicación de Resultados Genéticos.

En conclusión, es clave apoyar a los casos índice en la comunicación de la información de riesgo con sus familiares para obtener todos los beneficios de cuidado de la salud Genética. Los análisis de datos han demostrado que la difusión de la información dentro de las familias se fomenta de manera activa y apoyada por los profesionales. Surgen 3 temas principales: (1) El contacto directo de los servicios Genéticos: El envío de cartas a los familiares de portadores de la mutación; (2) El 
fomento profesional de los casos índice reacios a compartir información relevante con sus familiares en situación de riesgo; (3) La ayuda a los casos índice en la comunicación de la información genética a sus familiares en situación de riesgo, que incluyen como sub-temas: (i) La orientación psico-educativa; (ii) La información de ayuda por escrito. Los resultados sugieren que la práctica y las intervenciones de los profesionales se basan en la necesidad de fomentar de manera proactiva la comunicación familiar.

En este contexto, sería discutible si la orientación de los casos índice por profesionales podría ser apropiado, como buenas prácticas, a la hora de facilitar la comunicación familiar y serían discutibles los límites de la no-directividad en el Asesoramiento Genético ${ }^{(23)}$.

\section{Agradecimientos}

A todos los miembros de la Unidad de Asesoramiento Genético en Cáncer Hereditario del ICO. Estudio financiado en parte gracias al Fondo de Investigaciones Sanitarias P13/00189 y a la Junta de Barcelona de la Asociación Española contra el Cáncer.

\section{Referencias bibliográficas}

1. Andreu A, Balil J, Balmaña B, Bellosillo B, et al. Pràctica Clínica. OncoGuia del consell d'assessorament genètics en el càncer hereditàri. Barcelona: Agència d'Avaluació de Tecnologia i Recerca Mèdiques. Generalitat de Catalunya.; 2006.

2. Alonso A, Benavides MM, Blanco I, Brunet J, García-Foncillas J, Mayordomo JI, et al., editores. Cáncer hereditario II. Madrid: Sociedad Española de Oncología Médica; 2006.

3. Cabrera E, Blanco I, Yagüe C, Zabalegui A. The impact of genetic counseling on knowledge and emotional responses in Spanish population with family history of breast cancer. Patient Educ Couns 2010;78:382-8. Doi: 10.1016/j.pec.2009.10.032.

4. Koehly LM, Peters JA, Kenen R, Hoskins LM, Ersig AL, Kuhn NR, et al. Characteristics of health information gatherers, disseminators and blockers within families at risk of hereditary cancer: Implications for family health communication interventions. Am J Public Health 2009;99:2203-9. Doi: 10.2105/AJPH.2008.154096.

5. Stoffel EM, Ford B, Mercado RC, Punglia D, Kohlmann W, Conrad P, et al. Sharing genetic test results in Lynch syndrome: Communication with close and distant relatives. Clin Gastroenterol Hepatol 2008;6:333-8.

6. Koehly LM, Peterson SK, Watts BG, Kempf KK, Vernon SW, Gritz ER. A social network analysis of communication about hereditary nonpolyposis colorectal cancer genetic testing and family functioning. Cancer Epidemiol Biomarkers Prev 2003;12:304-13.

7. Tercyak KP, Peshkin BN, Demarco TA, Patenaude AF, Schneider KA, Garber JE, Valdimarsdottir HB, Schwartz MD. Information needs of mothers regarding communicating BRCA1/2 cancer genetic test results to their children. Genet Test 2007; 11(3):249-55.

8. Forrest LE, Burke J, Bacic S, Amor DJ. Increased genetic counseling support improves communication of genetic information in families. Genet Med 2008;10:167-72. Doi: 10.1097/GIM.0b013e318164540b.

9. Suthers GK, Armstrong J, McCormack J, Trott D. Letting the family know: Balancing ethics and effectiveness when notifying relatives about genetic testing for a familial disorder. J Med Genet 2006; 43:665-70. Doi: 10.1136/jmg.2005.039172 
10. Nycum G, Avard D, Knoppers BM. Factors influencing intrafamilial communication of hereditary breast and ovarian cancer genetic information. Eur J Hum Genet 2009; 17:87280. Doi: $10.1038 /$ ejhg.2009.33

11. Rothenberger DA, Dalberg DL, Leininger A. Minnesota Colorectal Cancer Initiative: Successful development and implementation of a community-based colorectal cancer registry. Dis Colon Rectum 2004; 47:1571-7. Doi: 10.1007/s10350-004-0649-1

12. González M, Blanco I, Blasco T. Efectos de la primera visita de asesoramiento genético sobre la percepción de riesgo y el malestar emocional. Psicooncología 2011; 8:441-51. Doi: 10.5209/rev_PSIC.2011.v8.n2-3.37891

13. González M, Blanco I, Bayes R. Dificultad en la comunicación del riesgo de padecer cáncer hereditario. Psicooncología 2005; 2:285-92.

14. Blanco I, Cabrera E, Llort G. Cáncer colorectal hereditario. Psicooncología 2005;2:213-28.

15. Medina-Arana V, Delgado L, González L, Bravo A, Díaz H, Salido E, et al. Adrenocortical carcinoma, an unusual extracolonic tumor associated with Lynch II syndrome. Fam Cancer 2011; 10:265-71. Doi: 10.1007/s10689-010-9416-8.

16. Ribeiro RC, Pinto EM, Zambetti GP. Familial predisposition to adrenocortical tumors: Clinical and biological features and management strategies. Best Pract Res Clin Endocrinol Metab 2010; 24:477-90. Doi: 10.1016/j.beem.2010.03.002.

17. Whyte S, Green A, McAllister M, Shipman H. Family Communication in inherited cardiovascular conditions in Ireland. J Genet Couns 2016;25:1317-26. Doi:10.1007/ s10897-016-9974-5

18. Mu PF, Lee MY, Sheng CC, Tung PC, Huang LY, Chen YW. The experiences of family members in the year following the diagnosis of a child or adolescent with cancer: A qualitative systematic review. JBI Database System Rev Implement Rep 2015: 13:293329. Doi: 10.11124/jbisrir-2015-1698329.

19. Raspa M, Edwards A, Wheeler AC, Bishop E, Bailey Jr. DB. Family communication and cascade testing for fragile X syndrome. J Genet Counsel 2016; 25:1075-84. Doi: 10.1007/ s10897-016-9940-2.

20. Heaton TJ, Chico V. Attitudes towards the sharing of genetic information with at-risk relatives: results of a quantitative survey. Hum Genet 2016;135:109-20. Doi: 10.1007/ s00439-015-1612-z.

21. Daly MB, Montgomery S, Bingler R, Ruth K. Communicating genetic test results within the family: Is it lost in translation? A survey of relatives in the randomized six-step study. Fam Cancer 2016;15:697-706. Doi: 10.1007/s10689-016-9889-1.

22. Elrick A, Ashida S, Ivanovich J, Lyons S, Biesecker BB, Goodman MS, et al. Psychosocial and clinical factors associated with family communication of cancer genetic test results among women diagnosed with breast cancer at a young age. J Genet Couns 2017 26:17381. Doi: 10.1007/s10897-016-9995-0

23. Mendes A, Paneque M, Sousa L, Clarke A, Sequeiros J. How communication of genetic information within the family is addressed in genetic counselling: A systematic review of research evidence. Eur J Hum Genet 2016;24:315-25. Doi: 10.1038/ejhg.2015.174 